Open Access

\title{
Pretargeting for imaging and therapy in oncological nuclear medicine
}

\author{
Clément Bailly ${ }^{1,3,4,5,6}$, Caroline Bodet-Milin ${ }^{1,3,4,5,6}$, Caroline Rousseau ${ }^{2,3,4,5,6}$, Alain Faivre-Chauvet 1,3,4,5,6, \\ Françoise Kraeber-Bodéré $1,2,3,4,5,6$ and Jacques Barbet ${ }^{6,7^{*}}$ (i)
}

\author{
* Correspondence: \\ jacques.barbet@nantes.inserm.fr \\ 'Université de Nantes, Nantes, \\ France \\ ${ }^{7}$ GIP Arronax, 1, rue Arronax, 44187 \\ Saint-Herblain cedex, France \\ Full list of author information is \\ available at the end of the article
}

\begin{abstract}
Background: Oncological pretargeting has been implemented and tested in several different ways in preclinical models and clinical trials over more than 30 years. Despite highly promising results, pretargeting has not achieved market approval even though it could be considered the ultimate theranostic, combining PET imaging with short-lived positron emitters and therapy with radionuclides emitting beta or alpha particles.

Results: We have reviewed the pretargeting approaches proposed over the years, discussing their suitability for imaging, particularly PET imaging, and therapy, as well as their limitations. The reviewed pretargeting modalities are the avidin-biotin system, bispecific anti-tumour $x$ anti-hapten antibodies and bivalent haptens, antibodyoligonucleotide conjugates and radiolabelled complementary oligonucleotides, and approaches using click chemistry. Finally, we discuss recent developments, such as the use of small binding proteins for pretargeting that may offer new perspectives to cancer pretargeting.
\end{abstract}

Conclusions: While pretargeting has shown promise and demonstrated preclinical and clinical proof of principle, full-scale clinical development programs are needed to translate pretargeting into a clinical reality that could ideally fit into current theranostic and precision medicine perspectives.

Keywords: Pretargeting, Immunoscintigraphy, ImmunoPET, Radioimmunotherapy, Bispecific antibody, Avidin-biotin, Oligonucleotides, Click chemistry

\section{Introduction}

Antibodies have been used as radionuclide carriers for imaging and therapywell before the discovery of monoclonal antibodies. The ability to produce unlimited amounts of homogenous products has prompted the development of antibodies for in vivo imaging and therapy, mostly in oncology. Contrary to the commercial and clinical success of naked therapeutic antibodies, only one labelled therapeutic antibody, Zevalin, for the treatment of B-cell lymphomas (Grillo-López 2002), and a few labelled antibodies for imaging (Goldenberg 1997) remain on the market. There are multiple reasons why labelled antibodies have not been commercially successful. Firstly, the pharmaceutical industry favours non-labelled products, as evidenced by the number of antibody-drug conjugates under development (Thomas et al. 2016), even though they do not outperform labelled antibodies in terms of efficacy or tolerance (Chatal et al. 2016). Secondly, referring physicians are reluctant to prescribe products they do not control.And finally,

(c) The Author(s). 2017 Open Access This article is distributed under the terms of the Creative Commons Attribution 4.0 International License (http://creativecommons.org/licenses/by/4.0/), which permits unrestricted use, distribution, and reproduction in any medium, provided you give appropriate credit to the original author(s) and the source, provide a link to the Creative Commons license, and indicate if changes were made. 
current therapeutic radiolabelled antibodies have a low therapeutic index..Indeed, radiolabelled antibodies in the form of intact IgG molecules, by far the most commonly used so far, have a very long persistence in the circulation, which can cause severe haematological side-effects. If tumour accretion may reach high levels (more than 10\% of injected activity per gram of tumour in mice and as much as $0.1 \%$ per gram of tumour in humans), this is only hours or days after activity injection and circulating activities remain elevated for days (Jain et al. 2007).

This explains why, in parallel to the development of therapeutic antibodies and labelled antibodies for tumour imaging, research on alternatives to intact IgG's has been very active. Antibody fragments and recombinant proteins, such as recombinant single chain Fv fragments, have been produced and tested. Whilst their blood clearance is faster, tumour accretion is reduced and kidney uptake is increased to levels inappropriate for therapy (Jain et al. 2007). Another alternative approach is pretargeting, whereby tumour target antigen binding specificity is obtained by injecting unlabelled antibody derivatives followed by a radiolabelled low molecular weight compound that specifically binds to the tumour and is rapidly cleared from the circulation (Goodwin et al. 1986). The goal is to achieve the targeting performances of the best peptides, such as somatostatin analogues, which have shown their efficacy in neuroendocrine tumours, both for imaging and for therapy (van Essen et al. 2014). Recent results with other low molecular weight compounds such as PSMA or CXCR4 inhibitors (Herrmann et al. 2016; Lütje et al. 2015; Vag et al. 2016) labelled with gallium-68 or fluorine-18 for PET imaging or lutetium- 177 for therapy, confirm that a low molecular weight and a fast clearance are important for efficient targeting and reduction of side effects. Nevertheless, because antibodies target a much wider range of antigens with potentially better specificity, they remain of high interest.

Goodwin et al. (Goodwin et al. 1986) and Halpern et al. (Halpern \& Dillman 1987) were the first to propose the use of a specific immunoconjugate recognizing both the target antigen and a low-molecular-weight substance carrying the radionuclide to detect or treat tumours. The unlabelled tumour-specific agent and the substance carrying the radionuclide were injected sequentially, with the radioactive compound being administered only after the antibody had localized in the target lesion and its excess was cleared, at least in part, from the circulation. This was first achieved using a bispecific antibody binding both a target antigen and a radiometal-chelate complex (Lollo et al. 1994; Stickney et al. 1991). Since then, many pretargeting studies have shown encouraging preclinical and clinical proof of concept results for both imaging and therapy (Goldenberg et al. 2006). Our historical review highlights these studies. However, after over thirty years of active research, no product has even come near to market approval. The possible reasons for this failure are also considered.

\section{Review}

\section{Early developments and the rationale of pretargeting}

In the early days of pretargeting, the main objective was tumour imaging. Indium-111 was the radionuclide of choice, and several pretargeting approaches were proposed (Goldenberg et al. 2006). Generally, antibodies or antibody fragments were used to recognize and bind the tumour cell target antigen, and a second agent was needed to bind the small molecule that carried the radioactive payload. Figure 1 schematizes a 


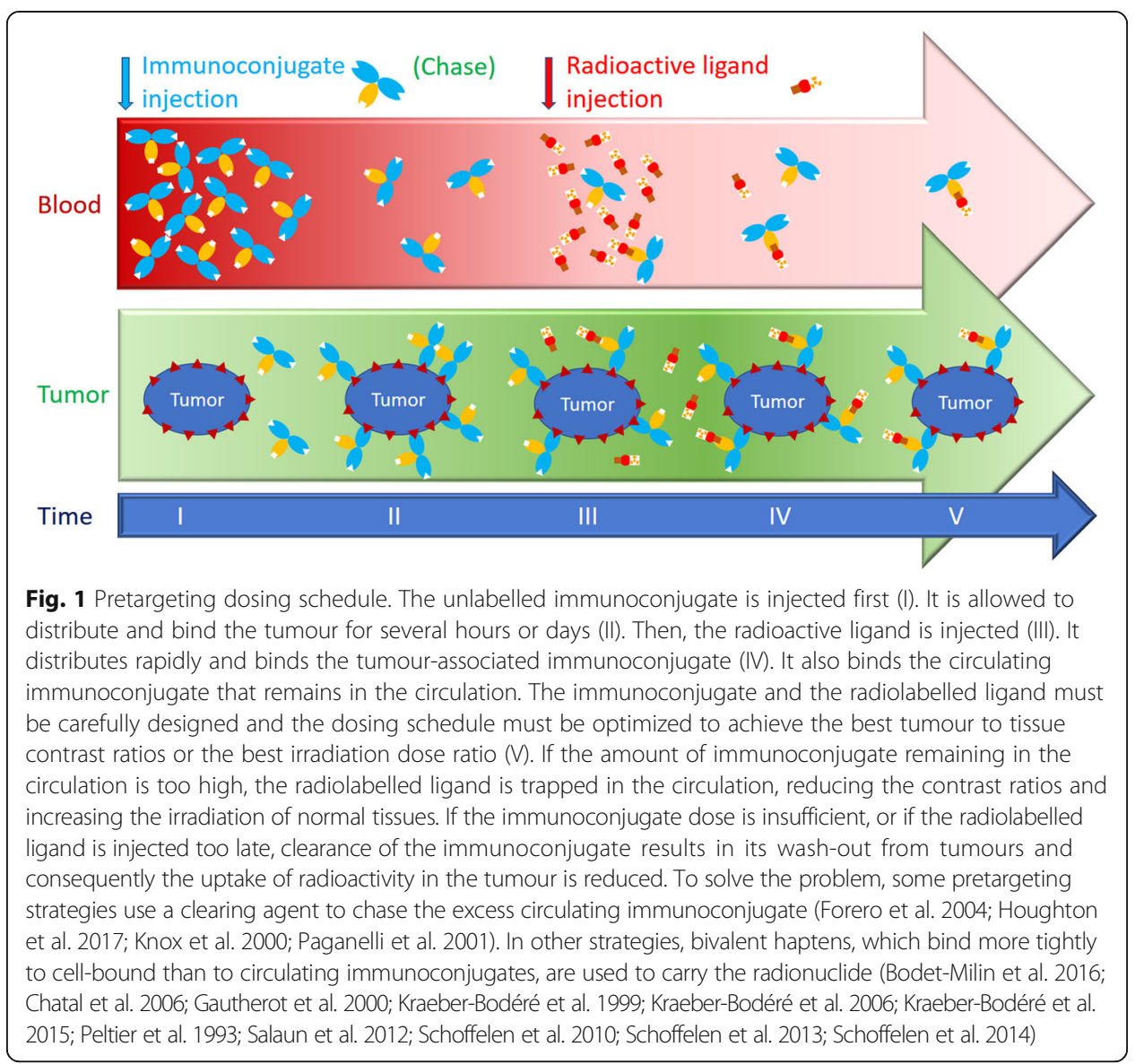

typical dosing schedule. Originally, the indium-benzyl-EDTA complex was used as a hapten, and bispecific antibodies binding carcinoembryonic antigen (CEA) and the hapten were used for imaging tumours (Lollo et al. 1994; Stickney et al. 1991). Biotin, which binds very tightly to avidin or streptavidin, was also proposed (Hnatowich et al. 1987; Pimm et al. 1988). The use of antibodies conjugated to an oligonucleotide binding to a radiolabelled complementary oligonucleotide was also proposed a few years later (Bos et al. 1994; Liu et al. 2002). Table 1 summarizes some of these approaches.

Whilst results were encouraging, low tumour uptake was observed if the injected dose of bispecific antibody was low or the delay between bispecific antibody and hapten injections was too long. Conversely, with a high dose of bispecific antibody and a short delay, tumour uptake was appreciable, but retention of the radioactive hapten in the circulation was high and protracted. Similar problems were observed using the avidinbiotin approach.There was clearly a need for significant improvements.

\section{Pretargeting with the avidin-biotin system}

With the avidin-biotin system, a chase step was proposed to remove a large fraction of the circulating antibody conjugate and subsequently reduce the background activity in normal tissues (Yao et al. 1995). However, by this time, the emphasis had shifted from tumour imaging to cancer therapy. Indeed, tumour imaging using antibodies is highly specific for target antigen expression and tumours not expressing the target antigen 


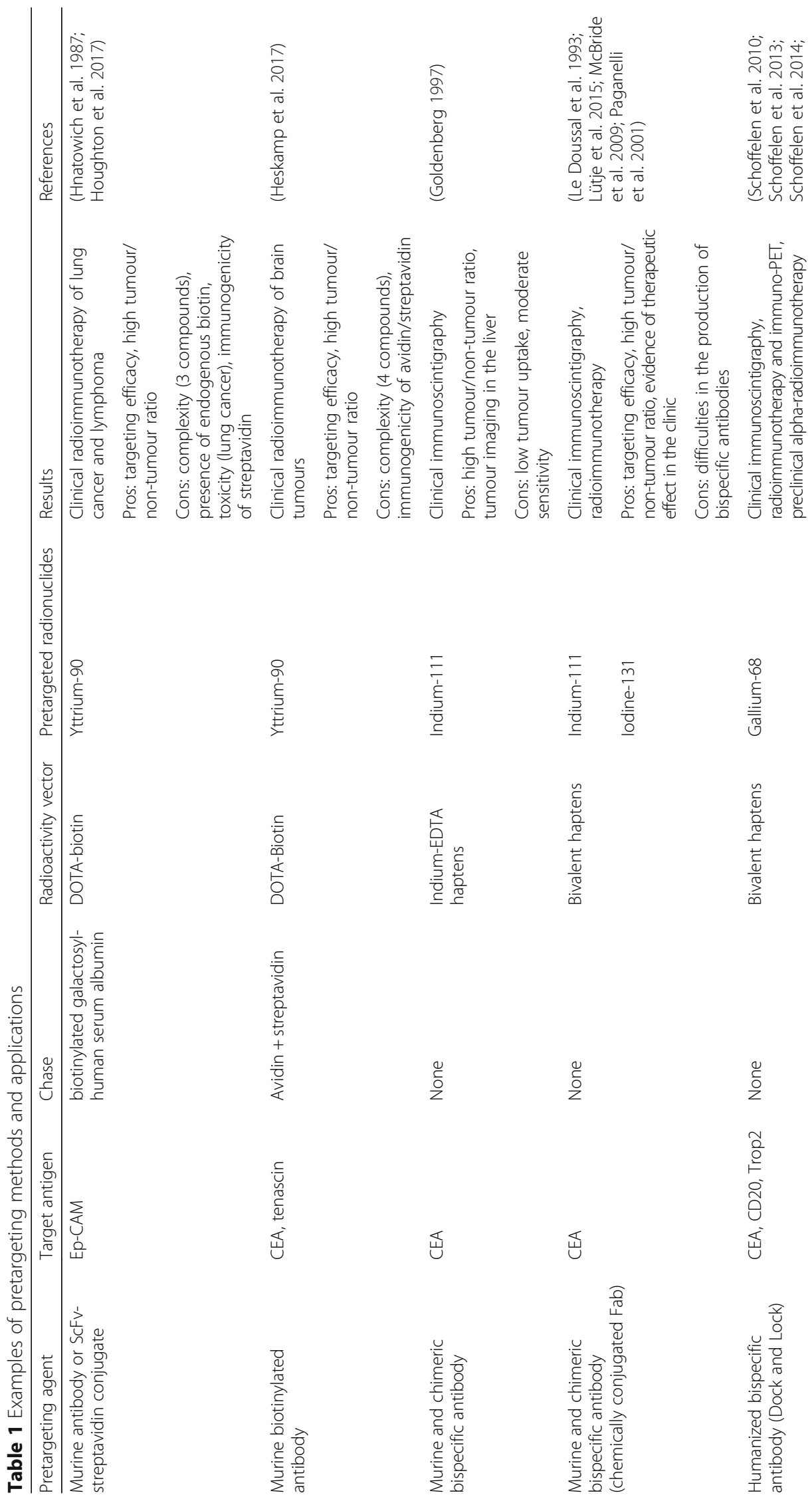




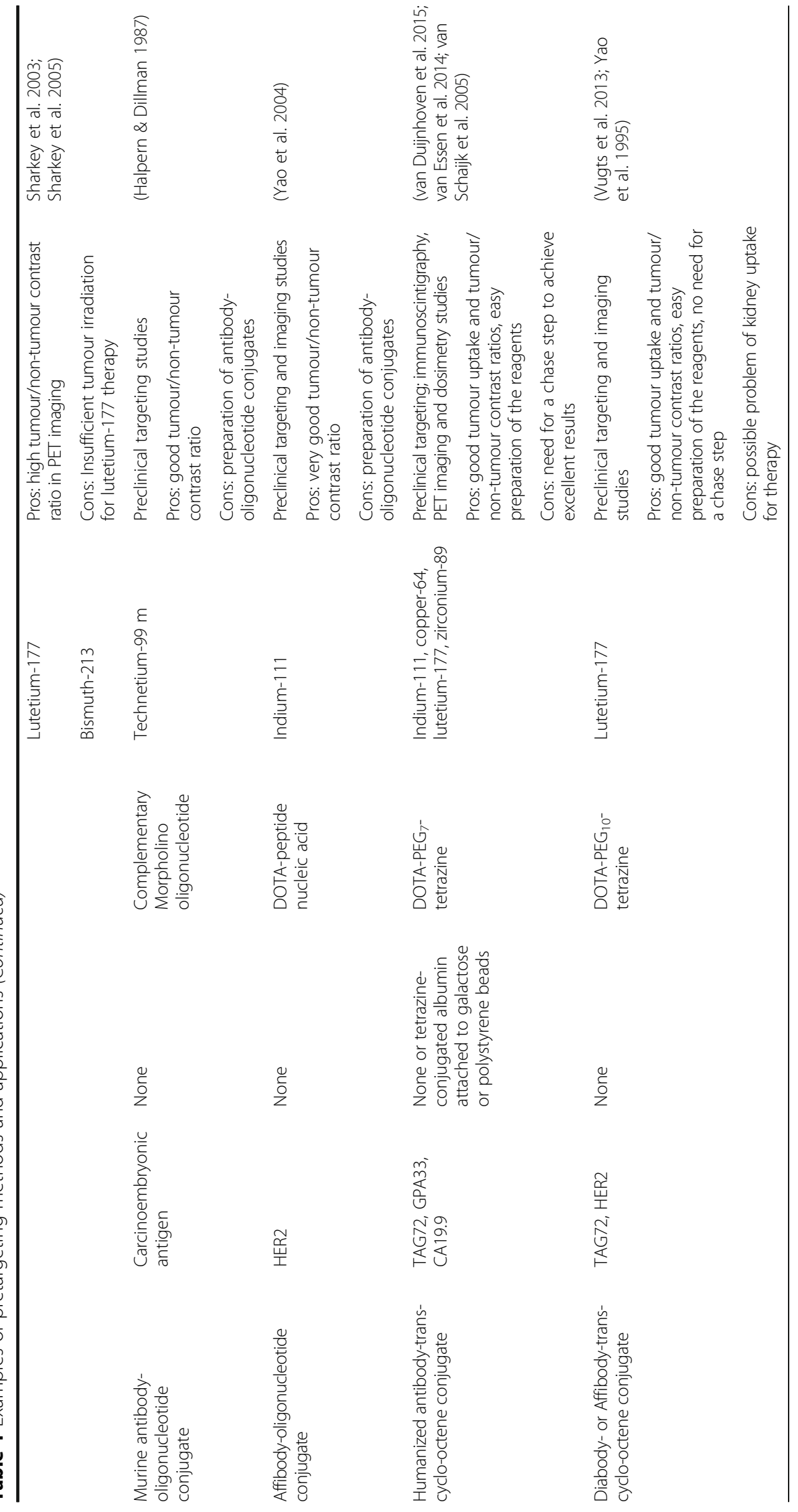


score as false-negatives in tumour detection. In addition, positron emission tomography (PET) with ${ }^{18} \mathrm{~F}$-fluoro-deoxy-glucose (FDG) had appeared as a general, sensitive, and sufficiently specific tumour detection method: immunoscintigraphy and pretargeted immunoscintigraphy appeared obsolete. However, pretargeting had been shown to be capable of delivering radiation doses to tumours, thus shifting the interest from tumour detection to tumour therapy.

Clinical studies were then performed using complex protocols involving three to five injections. Very good responses were obtained in patients with glioblastomas or anaplasic gliomas by Paganelli and co-workers using the sequential injection of (i) a biotinylated antibody (recognizing tenascin), (ii) avidin, to clear excess biotinylated antibody, (iii) streptavidin to decorate the tumour cell bound biotinylated antibody and (iv) yttrium-90-labelled DOTA-biotin (Paganelli et al. 2001). Twelve of the 48 treated patients benefited from $>25 \%$ tumour regression, and 8 of the 48 patients had a tumour response lasting for more than 12 months. The avidin-biotin system was also used for pretargeted radioimmunotherapy of lung cancer, but the lack of specificity of the targeting antibody resulted in severe toxicity (Knox et al. 2000). Pretargeting with the avidinbiotin system also showed promise in the treatment of lymphoma in both preclinical and clinical trials (Axworthy et al. 2000; Forero et al. 2004).

The advantage of the avidin-biotin system is that antibody-streptavidin or antibodybiotin conjugates are easily developed. In addition, available biotin derivatives may be readily radiolabelled. Although the published results are impressive, problems related to the immunogenicity of avidin and streptavidin have not been solved.

\section{Pretargeting with bispecific antibodies and radiolabelled haptens: The Affinity Enhancement System}

Bispecific antibodies may be prepared by a variety of techniques. In the early days, chemical conjugates of antibody fragments were used (Stickney et al. 1991) whereas hybrid hybridomas and quadromas were also considered (Kranenborg et al. 1995; van Schaijk et al. 2005). The presence of excess bispecific antibody in the circulation at the time of radiolabelled hapten injection was a problem, and resulted in relatively slow activity clearance and non-specific deposition in normal tissues. Increasing the time delay between the two injections resulted in poor tumour activity uptake. To circumvent this, Le Doussal and co-workers proposed the Affinity Enhancement System or AES (Le Doussal et al. 1990). This used a bivalent hapten that could cross-link bispecific antibody molecules at the surface of target cells and bind with enhanced affinity (or avidity), whereas, in the circulation, binding remained rapidly reversible, avoiding the need for a chaseof excess bispecific antibody. The interest of using bivalent haptens was independently confirmed by Goodwin and co-workers (Goodwin et al. 1992; Goodwin et al. 1994), who also proposed pretargeting for therapy using a monoclonal antibody raised against the yttrium-DOTA hapten, and later by Boerman and co-workers (Boerman et al. 1999), with a hybrid hybridoma of the anti-renal cell carcinoma antibody G250 and an antiDTPA-indium antibody.

In the original studies performed by our group in association with the French company Immunotech (Marseille, France), the bispecific antibody was prepared by chemical conjugation of the Fab fragment of the anti-CEA antibody F6 to the Fab fragment of an 
antibody recognizing the indium-DTPA complex. The bivalent hapten was prepared by reacting tyrosyl-lysine with DTPA anhydride. Surprisingly, the distance between the two indium-DTPA moieties may be quite short and the tyrosyl-lysine dipeptide, substituted by DTPA on both the $\alpha-\mathrm{NH}_{2}$ of tyrosine and the $\varepsilon-\mathrm{NH}_{2}$ of lysine, allows for the simultaneous in vitro binding of two anti-DTPA-indium antibodies (Le Doussal et al. 1990). This bivalent hapten could be labelled with indium-111, but also radio-

iodinated. However, the antibody specificity for the indium-DTPA complex limited radiolabelling to indium-111 or radioactive iodine. Bispecific antibodies recognizing another hapten, the histidine-succinyl-glycine (HSG) pseudo-peptide, were prepared and tested successfully with bivalent HSG haptens labelled with a variety of radionuclides (Janevik-Ivanovska et al. 1997).

\section{Pretargeted radioimmunodetection and pretargeted radioimmunotherapy (RIT) with bispecific antibodies and bivalent haptens (AES)}

Clinical AES pretargeted imaging using indium-111 scintigraphy produced high contrast images (Le Doussal et al. 1993; Peltier et al. 1993), particularly in medullary thyroid carcinoma (MTC), which consistently expresses CEA. However, successful tumour imaging required a long delay ( 2 to 4 days) between the bispecific antibody injection and injection of the labelled bivalent hapten, and high contrast images were obtained 24 hours later.The development of FDG-PET imaging further limited the interest of pretargeting even if the specificity was very high.

AES pretargeting could increase the radioimmunotherapy therapeutic index because it increases the tumour-to-normal tissue uptake ratio and increases radiation doses delivered to tumour cells. This was shown in preclinical models (Gautherot et al. 2000; Kraeber-Bodéré et al. 1999). The toxicity, pharmacokinetics, dosimetry and antitumour activity of the murine anti-CEA bispecific antibody F6x734 and the bivalent indium-DTPA hapten labelled with iodine-131 were evaluated in a phase I/II clinical trial in 26 patients with recurrent MTC (Kraeber-Bodéré et al. 2006). Haematological toxicity was the dose-limiting toxicity and the maximum tolerated activity was relatively low $\left(1.8 \mathrm{GBq} / \mathrm{m}^{2}\right)$. Whilst therapeutic responses were observed in only a small number of patients with small tumour burdens and after repeated courses of pretargeted RIT, long-term disease stabilization in a large number of the MTC patients (53\%) was documented by morphological imaging (computed tomography, MRI) and serial calcitonin and CEA measurements. The overall survival of 29 MTC patients treated by pretargeted RITA was compared to 39 contemporaneous untreated patients (Chatal et al. 2006). These patients were stratified according to calcitonin and CEA doubling times. Overall survival (OS) was significantly longer for high-risk treated patients (calcitonin Ct doubling time $<2$ years) compared to high-risk untreated patients (median OS, 110 vs. 61 months; $P<0.030$ ). Toxicity was mainly haematological, partly because of the frequent diffuse bone marrow tumour involvement. However, after treatment, patients with bone/bone marrow disease had a longer survival than patients without such involvement $(10$-year OS of $83 \%$ vs. $14 \% ; P<0.023)$. Interestingly, no other toxicity, especially renal toxicity, was reported. These results were confirmed in a prospective multicentric phase II trial. Treatment of progressive MTC patients (calcitonin doubling times shorter than 5 years) achieved a disease control rate (durable stabilization 
plus objective response) of $76.2 \%$ according to RECIST morphological imaging criteria, a durable complete response of more than 40 months in 1 patient (2.4\%) and durable stable disease ( $\geq 6$ months) in 31 patients (73.8\%) (Chatal et al. 2006). After RIT, 21 of 37 assessed patients (56.7\%) showed a $\geq 100 \%$ increase in calcitonin or CEA doubling times or prolonged decrease of the biomarker concentration. Hematologic toxicity (grade 3 and 4) was observed in $54.7 \%$ of the patients and myelodysplastic syndrome reported in 2 cases, including 1 previously heavily treated.

\section{Pretargeted PET imaging}

Recently, antibodies labelled with positron-emitters have been tested for PET imaging. Because of the slow pharmacokinetics of these antibodies, ong half-life positron emitters, such as zirconium-89 (half-life: 78.4 hours), were the first to be tested (Salaun et al. 2012). Antibodies labelled with copper-64 also provide good contrast images, but a shorter time interval is necessary because of the short radionuclide half-life ( 12.7 hours). Unfortunately the dosimetry is not very favourable, because zirconium- 89 emits a high-energy gamma photon and the copper-64 positron branching ratio is low (18\%). In addition, the imaging procedure cannot be performed within a single day after activity injection.

Pretargeting could improve the performance of immuno-PET and allow for the use of short half-life positron-emitting radionuclides such as gallium-68 or fluorine- 18 that would reduce patient irradiation. A few years ago Immunomedics Inc. developed the Dock-and-Lock $^{\mathrm{mm}}\left(\mathrm{DNL}^{\mathrm{mm}}\right)$ technology for producing humanized bispecific antibodies (Vugts et al. 2013). These consist of the regulatory subunits of cAMP-dependent protein kinase fused with one antibody Fab fragment, and the anchoring domains of A kinase fused with the other Fab fragment This allows a very efficient production of bispecific trivalent antibodies, with one site binding the hapten and two sites binding the tumour antigen. Several DNL ${ }^{\mathrm{m}}$ conjugates binding CEA (TF2), CD20 (TF4), a mucin antigen expressed by pancreatic tumours (TF10), and the Trop- 2 antigen (TF12) have been described (Gold et al. 2008; Rossi et al. 2006; Sharkey et al. 2005). In addition, using the histamine-succinyl-glycine (HSG) pseudo-peptide allowed construction of a new bivalent hapten, IMP288, permitting a variety of radionuclides to be used (Sharkey et al. 2012). Several studies showed that $\mathrm{DNL}^{\mathrm{mi}}$ bispecific antibodies were particularly well-suited to delivering short half-life radionuclides. In preclinical experiments, high contrast PET images could be obtained within an hour after radiolabelled hapten injection using gallium-68 and fluorine-18 (McBride et al. 2009; Sharkey et al. 2003). Extremely small tumours could be detected in mice. Pretargeted immuno-PET was then studied in the clinic for several cancers known to express CEA: breast cancers (Schoffelen et al. 2010), colorectal carcinoma and medullary thyroid cancers (Kraeber-Bodéré et al. 2015). Preliminary imaging results were very promising in the three tumours as shown for a breast cancer patient in Fig. 2. In all cases, pretargeted immuno-PET was able to detect very small tumour lesions after optimizing the TF2/IMP-288 molar dose ratio and the pretargeted delay for the antiCEA bispecific antibody TF2 and ${ }^{68}$ Ga-labeled IMP288 bivalent hapten. High tumour uptake was obtained with this approach in patients with relapsed MTC and HER2-negative breast cancer by injection of $120 \mathrm{nmol}$ of TF2 and $6 \mathrm{nmol}$ of ${ }^{68} \mathrm{Ga}-\mathrm{IMP}-288,30 \mathrm{~h}$ later. In some cases, immuno-PET allowed detection of lesions not detected by F-DOPA-PET, considered as the reference for PET imaging in MTC. 


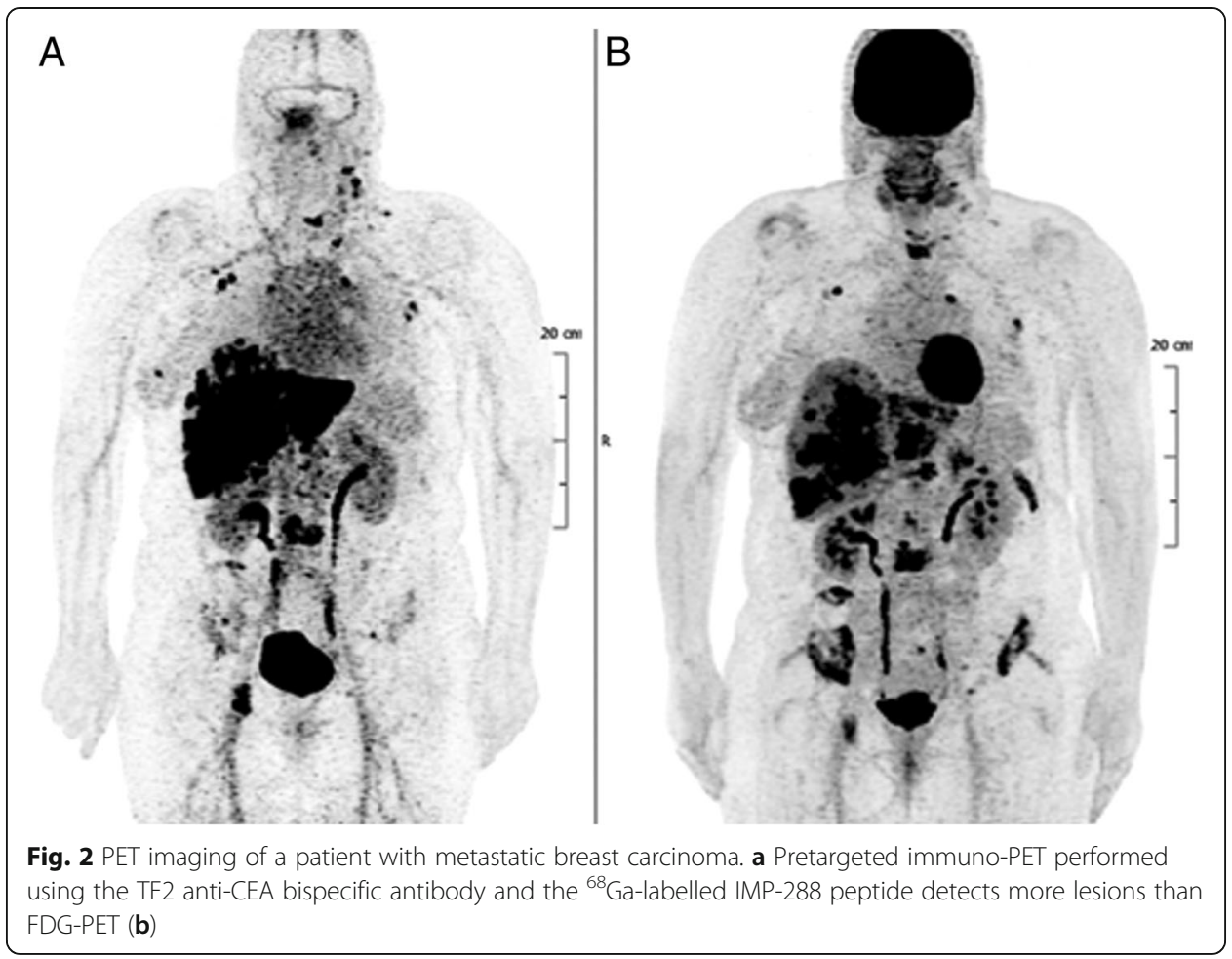

\section{Current research on pretargeting}

Based on the excellent PET imaging results using these new pretargeting agents, a clinical optimization studies were conducted to assess the anti-CEA $\times$ anti-HSG bispecific antibody TF2 and the radiolabelled hapten-peptide ${ }^{177} \mathrm{Lu}$-IMP288 in patients with metastatic CRC (Schoffelen et al. 2013). Different schedules were studied, and the best tumour targeting was achieved with a 1-day pretargeting interval, a high TF2 dose (150 mg) and a low $(25 \mu \mathrm{g})$ peptide dose. Rapid and selective tumour uptake was seen within $1 \mathrm{~h}$ after the peptide injection, with high tumour-to-tissue ratios at $24 \mathrm{~h}$. High activities of ${ }^{177} \mathrm{Lu}$-labelled IMP288 (2.5-7.4 GBq) were well tolerated, with some manageable reactions during the TF2 infusions and transient thrombocytopenia (grades $3-4)$ in $10 \%$ of the patients. Calculated radiation doses delivered to the kidneys and red bone marrow were relatively low (Schoffelen et al. 2014), allowing repeated administrations. Subsequently, two phase-I clinical trials were performed in France in patients with metastatic CEA-positive lung carcinoma using ${ }^{177} \mathrm{Lu}$-labelled IMP288, and in metastatic CRC patients using fractionated injections of TF2 and ${ }^{90}$ Y-IMP288. The therapeutic results were disappointing because of the relatively fast wash-out of tumour activity that limited tumour absorbed doses, especially with the long half-life lutetium-177.

Preclinical pharmacokinetic and dosimetry studies have also demonstrated that $\mathrm{DNL}^{\mathrm{m}}$ bispecific antibodies, because of their relatively fast blood clearance, could efficiently deliver short half-life radionuclides to tumours (Frampas et al. 2011). Short halflife radionuclides and particularly short half-life alpha-particle-emitting radionuclides, such as astatine-211 or bismuth-213, are considered for therapy, using both the avidinbiotin (Yao et al. 2004) and the AES approaches (Heskamp et al. 2017). 


\section{Alternative approaches in pretargeting}

In addition to the avidin-biotin and bispecific antibody approaches, two other alternatives have been proposed. The first relies on using the recognition between complementary DNA sequences. An antibody is derivatized with one oligonucleotide and the radioactivity is carried by an oligonucleotide of complementary sequence. This approach was proposed by Bos and co-workers (Bos et al. 1994) in 1994 and has not been very actively explored, despite the progress in the synthesis of oligonucleotides resistant to in vivo degradation. The other more recent approach is based on bio-orthogonal chemistry using the inverse-electron-demand Diels-Alder reaction between a tetrazine and a strained trans-cyclooctene (TCO) derivative. Rossin and co-workers first demonstrated that this reaction was fast enough and specific to be effective in vivo at the very low concentrations involved in cell targeting (Rossin et al. 2010; Rossin et al. 2013). In this seminal paper, a proof of concept of specific tumour uptake was achieved, but the tumour to normal tissue ratios were modest (Rossin et al. 2010): when an intact IgG is used to prepare the pretargeting immunoconjugate, the presence of excess circulating immunoconjugate at the time of injection of the click-chemistry partner reacts with the labelled molecule and reduces its clearance, as observed with the other pretargeting systems many years earlier. Similar biodistribution results were obtained in another system that also showed positive PET imaging (Zeglis et al. 2013). In a recent paper, Hougton et al. evaluated the click chemistry approach for therapy. Whilst the activity accretion in tumours was high the circulating activity was also high and declined slowly. To solve this problem, Rossin and co-workers used a chase step that considerably improved tumour uptake and tumour to non-tumour ratios (Houghton et al. 2017). As with the avidin-biotin system, the use of a chase step could therefore be a solution, but would be rather cumbersome with a risk of immunogenicity and hypersensitivity side-effects.

The click chemistry pretargeting approach has been tested with a fast clearing antibody derivative: a $60 \mathrm{kDa}$ diabody (van Duijnhoven et al. 2015). High tumour uptake (6.9\% injected dose/g) and tumour to blood ratios were observed, with reasonable kidney uptake, and without a chase step demonstrating that faster immunconjugate clearance could be a solution. Tolmachev and co-workers went a step further by using an Affibody, a very small binding protein of $7 \mathrm{kDa}$, as pretargeting agent (Altai et al. 2016). Tumour accretion and clearance of the pretargeting agent were fast enough to establish a large difference between the amount of Affibody bound to the tumour and that remaining in the circulation. The same Affibody was also used with very good pretargeting results using the oligonucleotide (or peptide nucleotide in that case) approach (Honarvar et al. 2016). Further studies will show whether this promising approach can overcome the current limitations of pretargeting.

\section{Conclusions and perspectives}

Pretargeting originated in the mid-80s, and has been implemented in several different ways and tested in preclinical models and clinical trials. Despite highly promising results in preclinical tumour models, as well as in early phase clinical trials, pretargeting has not yet come close to market approval. However, pretargeting remains as potentially the ultimate theranostic, combining PET imaging with short-lived positron emitters and therapy with radionuclides emitting beta or alpha particles. Producing the 
required humanized recombinant immunoconjugates remains a challenge. Quadromas and chemically-coupled bispecific antibodies are difficult and expensive to prepare under GMP conditions and may not have the required purity for clinical development. $\mathrm{DNL}^{\mathrm{TM}}$ bispecific antibodies proved very efficient for imaging, but not as effective for therapy. Bispecific diabodies and triabodies that appear very appropriate in terms of molecular weight and stability (Rossi et al. 2005) have not been produced in the necessary mass quantities so far. Adaptation of older approaches that use avidin biotin or bispecific anti-tumour $\mathrm{x}$ anti-hapten antibodies with pretargeting using in vivo click chemistry show promise. The use of small binding proteins for pretargeting may also offer a new perspective. Indeed, these binding proteins have fast in vivo kinetics, but tend to deliver very high activity to the kidneys (as do most peptides), when radiolabelled with metal radionuclides. Pretargeting could be a way to overcome the problem and provide a new targeting approach for both imaging and therapy. Pretargeting is also one way to use short-lived radionuclides to image and treat cancers that do not over-express receptors or enzymes that could be targeted with small peptides or inhibitors.

In conclusion, pretargeting remains quite attractive, particularly for PET imaging and therapy, in a theranostic perspective. However, it requires careful optimization, both for the design of the appropriate pretargeting reagents, bispecific immunoconjugates binding tumour antigens and the small molecule selected to carry the activity, and for the definition of dosing and administration schedules. Full-scale clinical development programs remain needed to translate pretargeting into a clinical reality.

\section{Abbreviations}

CEA: Carcinoembryonic antigen; DNL ${ }^{\mathrm{TM}}$ : Dock-and-Lock ${ }^{\mathrm{TM}}$; DTPA: Diethylene triamine pentaacetic acid; FDG: ${ }^{18} \mathrm{~F}-f l u o r o-$ deoxy-glucose; F-DOPA: L-3,4-Dihydroxy-6- $\left[{ }^{18} \mathrm{~F}\right]$ fluorophenylalanine; HSG: Histidine-succinyl-glycine; MTC: Medullary thyroid carcinoma; OS: Overall survival; PET: Positron emission tomography; RIT: Radioimmunotherapy.

\section{Acknowledgments}

This work has been supported in part by grants from the French National Agency for Research, called "Investissements d'Avenir" IRON Labex n ANR-11-LABX-0018-01 and ArronaxPlus Equipex n ANR-11-EQPX-0004.

The authors thank D.M. Goldenberg (Immunomedics, Inc. Morris Plains, NJ, USA) for continuous support.

\section{Funding}

This work was funded by grants of the French National Agency for Research within the "Investissements d'Avenir" program: Equipex ArronaxPlus (ANR-11-EQPX-0004) and Labex IRON (ANR-11-LABX-0018).

Authors' contributions

All authors participated in writing the manuscript. All authors read and approved the final manuscript.

Competing interests

The authors declare that they have no competing interests.

Consent for publication

Not applicable.

\section{Ethics approval and consent to participate}

This paper reviews previously published data about preclinical animal studies and clinical trials, thus ethics approval and consent to participate are not applicable. Figure 2 shows an image from a clinical trial, sponsored by Nantes University Hospital, that was approved by the responsible ethics committee (CPP), registered at ClinicalTrial.gov (NCT01730612), and all patients signed a written informed consent form.

\section{Publisher's Note}

Springer Nature remains neutral with regard to jurisdictional claims in published maps and institutional affiliations.

\section{Author details}

${ }^{1}$ Service de Médecine Nucléaire, CHU de Nantes, Nantes, France. ${ }^{2}$ Service de Médecine Nucléaire, Institut de Cancérologie de l'Ouest, Saint-Herblain, France. ${ }^{3}$ Centre de Recherche en Cancérologie Nantes/Angers (CRCNA), Nantes, France. ${ }^{4} 6299$ CNRS, Nantes, France. ${ }^{5}$ UMR892 Inserm, Nantes, France. ${ }^{6}$ Université de Nantes, Nantes, France. ${ }^{7}$ GIP Arronax, 1, rue Arronax, 44187 Saint-Herblain cedex, France. 
Received: 16 March 2017 Accepted: 24 May 2017

Published online: 06 June 2017

\section{References}

Altai M, Perols A, Tsourma M, Mitran B, Honarvar H, Robillard M, Rossin R, ten Hoeve W, Lubberink M, Orlova A, Karlström AE, Tolmachev V. Feasibility of Affibody-Based Bioorthogonal Chemistry-Mediated Radionuclide Pretargeting. J Nucl Med. 2016;57:431-6.

Axworthy DB, Reno JM, Hylarides MD, Mallett RW, Theodore LJ, Gustavson LM, Su F, Hobson L, Beaumier PL, Fritzberg AR. Cure of human carcinoma xenografts by a single dose of pretargeted yttrium-90 with negligible toxicity. Proc Natl Acad Sci U S A. 2000;97:1802-7.

Bodet-Milin C, Faivre-Chauvet A, Carlier T, Rauscher A, Bourgeois M, Cerato E, Rohmer V, Couturier O, Drui D, Goldenberg DM, Sharkey RM, Barbet J, Kraeber-Bodere F. Immuno-PET Using Anticarcinoembryonic Antigen Bispecific Antibody and 68Ga-Labeled Peptide in Metastatic Medullary Thyroid Carcinoma: Clinical Optimization of the Pretargeting Parameters in a First-in-Human Trial. J Nucl Med. 2016;57:1505-11.

Boerman OC, Kranenborg MH, Oosterwijk E, Griffiths GL, McBride WJ, Oyen WJ, de Weijert M, Oosterwijk-Wakka J, Hansen HJ, Corstens FH. Pretargeting of renal cell carcinoma: improved tumor targeting with a bivalent chelate. Cancer Res. 1999:59:4400-5.

Bos ES, Kuijpers WH, Meesters-Winters M, Pham DT, de Haan AS, van Doornmalen AM, Kaspersen FM, van Boeckel CA, Gougeon-Bertrand F. In vitro evaluation of DNA-DNA hybridization as a two-step approach in radioimmunotherapy of cancer. Cancer Res. 1994;54:3479-86.

Chatal JF, Campion L, Kraeber-Bodéré F, Bardet S, Vuillez JP, Charbonnel B, Rohmer V, Chang CH, Sharkey RM, Goldenberg DM, Barbet J, French Endocrine Tumor Group. Survival improvement in patients with medullary thyroid carcinoma who undergo pretargeted anti-carcinoembryonic-antigen radioimmunotherapy: a collaborative study with the French Endocrine Tumor Group. J Clin Oncol. 2006;24:1705-11.

Chatal JF, Kraeber-Bodéré F, Bodet-Milin C, Rousseau C. Therapeutic Immunoconjugates. Which Cytotoxic Payload: Chemotherapeutic Drug (ADC) or Radionuclide (ARC)? Current Cancer Ther Rev. 2016;12:54-65.

Forero A, Weiden PL, Vose JM, Knox SJ, LoBuglio AF, Hankins J, Goris ML, Picozzi VJ, Axworthy DB, Breitz HB, Sims RB, Ghalie RG, Shen S, Meredith RF. Phase 1 trial of a novel anti-CD20 fusion protein in pretargeted radioimmunotherapy for B-cell non-Hodgkin lymphoma. Blood. 2004;104:227-36.

Frampas E, Maurel C, Remaud-Le Saëc P, Mauxion T, Faivre-Chauvet A, Davodeau F, Goldenberg DM, Bardiès M, Barbet J. Pretargeted radioimmunotherapy of colorectal cancer metastases: models and pharmacokinetics predict influence of the physical and radiochemical properties of the radionuclide. Eur J Nucl Med Mol Imaging. 2011;38:2153-64.

Gautherot E, Rouvier E, Daniel L, Loucif E, Bouhou J, Manetti C, Martin M, Le Doussal JM, Barbet J. Pretargeted radioimmunotherapy of human colorectal xenografts with bispecific antibody and ${ }^{131}$-labeled bivalent hapten. J Nucl Med. 2000;41:480-7.

Gold DV, Goldenberg DM, Karacay H, Rossi EA, Chang CH, Cardillo TM, McBride WJ, Sharkey RM. A novel bispecific, trivalent antibody construct for targeting pancreatic carcinoma. Cancer Res. 2008;68:4819-26.

Goldenberg DM. Perspectives on oncologic imaging with radiolabeled antibodies. Cancer. 1997;80:2431-5.

Goldenberg DM, Sharkey RM, Paganelli G, Barbet J, Chatal JF. Antibody pretargeting advances cancer radioimmunodetection and radioimmunotherapy. J Clin Oncol. 2006;24:823-34.

Goodwin DA, Meares CF, David GF, McTigue M, McCall MJ, Frincke JM, Stone MR, Bartholomew RM, Leung JP. Monoclonal antibodies as reversible equilibrium carriers of radiopharmaceuticals. Int J Rad Appl Instrum B. 1986;13:383-91.

Goodwin DA, Meares CF, McTigue M, Chaovapong W, Diamanti Cl, Ransone CH, McCall MJ. Pretargeted immunoscintigraphy: effect of hapten valency on murine tumor uptake. J Nucl Med. 1992;33:2006-13.

Goodwin DA, Meares CF, Watanabe N, McTigue M, Chaovapong W, Ransone CM, Renn O, Greiner DP, Kukis DL, Kronenberger SI. Pharmacokinetics of pretargeted monoclonal antibody 2D12.5 and 88Y-Janus-2-(p-nitrobenzy)-1,4,7,10tetraazacyclododecanetetraacetic acid (DOTA) in BALB/C mice with KHJJ mouse adenocarcinoma: a model for 90Y radioimmunotherapy. Cancer Res. 1994;54:5937-46.

Grillo-López AJ. Zevalin: the first radioimmunotherapy approved for the treatment of lymphoma. Expert Rev Anticancer Ther. 2002;2:485-93.

Halpern SE, Dillman RO. Problems associated with radioimmunodetection and possibilities for future solutions. J Biol Response Mod. 1987;6:235-62.

Herrmann K, Schottelius M, Lapa C, OsI T, Poschenrieder A, Hänscheid H, Lückerath K, Schreder M, Bluemel C, Knott M, Keller U, Schirbel A, Samnick S, Lassmann M, Kropf S, Buck AK, Einsele H, Wester HJ, Knop S. First-in-Human Experience of CXCR4-Directed Endoradiotherapy with ${ }^{177} \mathrm{Lu}$ - and ${ }^{90}$ Y-Labeled Pentixather in Advanced-Stage Multiple Myeloma with Extensive Intra- and Extramedullary Disease. J Nucl Med. 2016;57:248-51.

Heskamp S, Hernandez R, Molkenboer-Kuenen JD, Essler M, Bruchertseifer F, Morgenstern A, Steenbergen E, Cai W, Seidl C, McBride W, Goldenberg D, Boerman O. Alpha- versus beta-emitting radionuclides for pretargeted radioimmunotherapy of CEA-expressing human colon cancer xenografts. J Nucl Med. 2017. in press.

Hnatowich DJ, Virzi F, Rusckowski M. Investigations of avidin and biotin for imaging applications. J Nucl Med. 1987;28:1294-302.

Honarvar H, Westerlund K, Altai M, Sandström M, Orlova A, Tolmachev V, Karlström AE. Feasibility of Affibody MoleculeBased PNA-Mediated Radionuclide Pretargeting of Malignant Tumors. Theranostics. 2016;6:93-103.

Houghton JL, Membreno R, Abdel-Atti D, Cunanan KM, Carlin S, Scholz WW, Zanzonico PB, Lewis JS, Zeglis BM. Establishment of the In Vivo Efficacy of Pretargeted Radioimmunotherapy Utilizing Inverse Electron Demand DielsAlder Click Chemistry. Mol Cancer Ther. 2017;16:124-33.

Jain M, Venkatraman G, Batra SK. Optimization of radioimmunotherapy of solid tumors: biological impediments and their modulation. Clin Cancer Res. 2007;13:1374-82.

Janevik--vanovska E, Gautherot E, Hillairet de Boisferon M, Cohen M, Milhaud G, Tartar A, Rostene W, Barbet J, Gruaz-Guyon A. Bivalent hapten-bearing peptides designed for iodine-131 pretargeted radioimmunotherapy. Bioconjug Chem. 1997;8:526-33.

Knox SJ, Goris ML, Tempero M, Weiden PL, Gentner L, Breitz H, Adams GP, Axworthy D, Gaffigan S, Bryan K, Fisher DR, Colcher D, Horak ID, Weiner LM. Phase II trial of yttrium-90-DOTA-biotin pretargeted by NR-LU-10 antibody/ streptavidin in patients with metastatic colon cancer. Clin Cancer Res. 2000;6:406-14. 
Kraeber-Bodéré F, Faibre-Chauvet A, Saï-Maurel C, Gautherot E, Fiche M, Campion L, Le Boterff J, Barbet J, Chatal JF, Thédrez P. Bispecific antibody and bivalent hapten radioimmunotherapy in CEA-producing medullary thyroid cancer xenograft. J Nucl Med. 1999;40:198-204.

Kraeber-Bodéré F, Rousseau C, Bodet-Milin C, Ferrer L, Faivre-Chauvet A, Campion L, Vuillez JP, Devillers A, Chang CH, Goldenberg DM, Chatal JF, Barbet J. Targeting, toxicity, and efficacy of 2-step, pretargeted radioimmunotherapy using a chimeric bispecific antibody and ${ }^{131}$ I-labeled bivalent hapten in a phase I optimization clinical trial. J Nucl Med. 2006;47:247-55.

Kraeber-Bodéré F, Rousseau C, Bodet-Milin C, Frampas E, Faivre-Chauvet A, Rauscher A, Sharkey RM, Goldenberg DM, Chatal JF, Barbet J. A pretargeting system for tumor PET imaging and radioimmunotherapy. Front Pharmacol. 2015;6:54.

Kranenborg MH, Boerman OC, Oosterwijk-Wakka JC, de Weijert MC, Corstens FH, Oosterwijk E. Development and characterization of anti-renal cell carcinoma $x$ antichelate bispecific monoclonal antibodies for two-phase targeting of renal cell carcinoma. Cancer Res. 1995;55:5864s-7s.

Le Doussal JM, Gruaz-Guyon A, Martin M, Gautherot E, Delaage M, Barbet J. Targeting of indium 111-labeled bivalent hapten to human melanoma mediated by bispecific monoclonal antibody conjugates: imaging of tumors hosted in nude mice. Cancer Res. 1990;50:3445-52. Erratum in: Cancer Res 1990;50:6115.

Le Doussal JM, Chetanneau A, Gruaz-Guyon A, Martin M, Gautherot E, Lehur PA, Chatal JF, Delaage M, Barbet J. Bispecific monoclonal antibody-mediated targeting of an indium-111-labeled DTPA dimer to primary colorectal tumors: pharmacokinetics, biodistribution, scintigraphy and immune response. J Nucl Med. 1993;34:1662-71.

Liu G, Mang'era K, Liu N, Gupta S, Rusckowski M, Hnatowich DJ. Tumor pretargeting in mice using ${ }^{99 \mathrm{~m}}$ Tc-labeled morpholino, a DNA analog. J Nucl Med. 2002;43:384-91.

Lollo C, Halpern S, Bartholomew R, David G, Hagan P. Non-covalent antibody-mediated drug delivery. Nucl Med Commun. 1994;15:483-91.

Lütje S, Heskamp S, Cornelissen AS, Poeppel TD, van den Broek SA, Rosenbaum-Krumme S, Bockisch A, Gotthardt M, Rijpkema M, Boerman OC. PSMA Ligands for Radionuclide Imaging and Therapy of Prostate Cancer: Clinical Status. Theranostics. 2015;5:1388-401.

McBride WJ, Sharkey RM, Karacay H, D'Souza CA, Rossi EA, Laverman P, Chang CH, Boerman OC, Goldenberg DM. A novel method of ${ }^{18} \mathrm{~F}$ radiolabeling for PET. J Nucl Med. 2009;50:991-8.

Paganelli G, Bartolomei M, Ferrari M, Cremonesi M, Broggi G, Maira G, Sturiale C, Grana C, Prisco G, Gatti M, Caliceti P, Chinol M. Pre-targeted locoregional radioimmunotherapy with 90Y-biotin in glioma patients: phase I study and preliminary therapeutic results. Cancer Biother Radiopharm. 2001;16:227-35.

Peltier P, Curtet C, Chatal JF, Le Doussal JM, Daniel G, Aillet G, Gruaz-Guyon A, Barbet J, Delaage M. Radioimmunodetection of medullary thyroid cancer using a bispecific anti-CEAvanti-indium-DTPA antibody and an indium-111-labeled DTPA dimer. J Nucl Med. 1993;34:1267-73.

Pimm MV, Fells HF, Perkins AC, Baldwin RW. lodine-131 and indium-111 labelled avidin and streptavidin for pre-targetted immunoscintigraphy with biotinylated anti-tumour monoclonal antibody. Nucl Med Commun. 1988;9:931-41.

Rossi EA, Chang CH, Losman MJ, Sharkey RM, Karacay H, McBride W, Cardillo TM, Hansen HJ, Qu Z, Horak ID, Goldenberg DM. Pretargeting of carcinoembryonic antigen-expressing cancers with a trivalent bispecific fusion protein produced in myeloma cells. Clin Cancer Res. 2005;11:7122s-9s.

Rossi EA, Goldenberg DM, Cardillo TM, McBride WJ, Sharkey RM, Chang CH. Stably tethered multifunctional structures of defined composition made by the dock and lock method for use in cancer targeting. Proc Natl Acad Sci U S A. 2006;103:6841-6.

Rossin R, Verkerk PR, van den Bosch SM, Vulders RC, Verel I, Lub J, Robillard MS. In vivo chemistry for pretargeted tumor imaging in live mice. Angew Chem Int Ed Engl. 2010;49:3375-8.

Rossin R, Läppchen T, van den Bosch SM, Laforest R, Robillard MS. Diels-Alder reaction for tumor pretargeting: in vivo chemistry can boost tumor radiation dose compared with directly labeled antibody. J Nucl Med. 2013;54:1989-95.

Salaun PY, Campion L, Bournaud C, Faivre-Chauvet A, Vuillez JP, Taieb D, Ansquer C, Rousseau C, Borson-Chazot F, Bardet S, Oudoux A, Cariou B, Mirallié E, Chang CH, Sharkey RM, Goldenberg DM, Chatal JF, Barbet J, KraeberBodéré F. Phase II trial of anticarcinoembryonic antigen pretargeted radioimmunotherapy in progressive metastatic medullary thyroid carcinoma: biomarker response and survival improvement. J Nucl Med. 2012;53:1185-92.

Schoffelen R, Sharkey RM, Goldenberg DM, Franssen G, McBride WJ, Rossi EA, Chang CH, Laverman P, Disselhorst JA, Eek A, van der Graaf WT, Oyen WJ, Boerman OC. Pretargeted immuno-positron emission tomography imaging of carcinoembryonic antigen-expressing tumors with a bispecific antibody and a ${ }^{68} \mathrm{Ga}$ - and ${ }^{18} \mathrm{~F}$-labeled hapten peptide in mice with human tumor xenografts. Mol Cancer Ther. 2010;9:1019-27.

Schoffelen R, Boerman OC, Goldenberg DM, Sharkey RM, van Herpen CM, Franssen GM, et al. Development of an imaging-guided CEA-pretargeted radionuclide treatment of advanced colorectal cancer: first clinical results. Br J Cancer. 2013;109:934-42.

Schoffelen R, Woliner-van der Weg W, Visser EP, Goldenberg DM, Sharkey RM, McBride WJ, Chang CH, Rossi EA, van der Graaf WT, Oyen WJ, Boerman OC. Predictive patient-specific dosimetry and individualized dosing of pretargeted radioimmunotherapy in patients with advanced colorectal cancer. Eur J Nucl Med Mol Imaging. 2014;41:1593-602.

Sharkey RM, McBride WJ, Karacay H, Chang K, Griffiths GL, Hansen HJ, Goldenberg DM. A universal pretargeting system for cancer detection and therapy using bispecific antibody. Cancer Res. 2003;63:354-63.

Sharkey RM, Karacay H, Chang CH, McBride WJ, Horak ID, Goldenberg DM. Improved therapy of non-Hodgkin's lymphoma xenografts using radionuclides pretargeted with a new anti-CD20 bispecific antibody. Leukemia. 2005;19:1064-9.

Sharkey RM, van Rij CM, Karacay H, Rossi EA, Frielink C, Regino C, Cardillo TM, McBride WJ, Chang CH, Boerman OC, Goldenberg DM. A new Tri-Fab bispecific antibody for pretargeting Trop-2-expressing epithelial cancers. J Nucl Med. 2012;53:1625-32

Stickney DR, Anderson LD, Slater JB, Ahlem CN, Kirk GA, Schweighardt SA, Frincke JM. Bifunctional antibody: a binary radiopharmaceutical delivery system for imaging colorectal carcinoma. Cancer Res. 1991;51:6650-5.

Thomas A, Teicher BA, Hassan R. Antibody-drug conjugates for cancer therapy. Lancet Oncol. 2016;17:e254-62.

Vag T, Gerngross C, Herhaus P, Eiber M, Philipp-Abbrederis K, Graner FP, Ettl J, Keller U, Wester HJ, Schwaiger M. First Experience with Chemokine Receptor CXCR4-Targeted PET Imaging of Patients with Solid Cancers. J Nucl Med. 2016;57:741-6.

van Duijnhoven SM, Rossin R, van den Bosch SM, Wheatcroft MP, Hudson PJ, Robillard MS. Diabody Pretargeting with Click Chemistry In Vivo. J Nucl Med. 2015;56:1422-8. 
van Essen M, Sundin A, Krenning EP, Kwekkeboom DJ. Neuroendocrine tumours: the role of imaging for diagnosis and therapy. Nat Rev Endocrinol. 2014;10:102-14.

van Schaijk FG, Oosterwijk E, Soede AC, Broekema M, Frielink C, McBride WJ, Goldenberg DM, Corstens FH, Boerman OC. Pretargeting of carcinoembryonic antigen-expressing tumors with a biologically produced bispecific anticarcinoembryonic antigen $\mathrm{x}$ anti-indium-labeled diethylenetriaminepentaacetic acid antibody. Clin Cancer Res. 2005;11:7130s-6s.

Vugts DJ, Visser GW, van Dongen GA. ${ }^{89} \mathrm{Zr}$-PET radiochemistry in the development and application of therapeutic monoclonal antibodies and other biologicals. Curr Top Med Chem. 2013;13:446-57.

Yao Z, Zhang M, Kobayashi H, Sakahara H, Nakada H, Yamashina I, Konishi J. Improved targeting of radiolabeled streptavidin in tumors pretargeted with biotinylated monoclonal antibodies through an avidin chase. J Nucl Med. 1995;36:837-41.

Yao Z, Zhang M, Garmestani K, Axworthy DB, Mallett RW, Fritzberg AR, Theodore L, Plascjak PS, Eckelman WC, Waldmann TA, Pastan I, Paik CH, Brechbiel MW, Carrasquillo JA. Pretargeted alpha emitting radioimmunotherapy using 213Bi 1,4,7,10-tetraazacyclododecane-N, N', N", N"'-tetraacetic acid-biotin. Clin Cancer Res. 2004;10:3137-46.

Zeglis BM, Sevak KK, Reiner T, Mohindra P, Carlin SD, Zanzonico P, Weissleder R, Lewis JS. A pretargeted PET imaging strategy based on bioorthogonal Diels-Alder click chemistry. J Nucl Med. 2013;54:1389-96.

Submit your manuscript to a SpringerOpen ${ }^{\circ}$ journal and benefit from:

- Convenient online submission

- Rigorous peer review

- Open access: articles freely available online

- High visibility within the field

- Retaining the copyright to your article

Submit your next manuscript at $\gg$ springeropen.com 